\title{
The Proton Radius Puzzle - A Challenge To All Of Us
}

\author{
Gerald A. Miller*i \\ University of Washington, USA \\ E-mail: miller@phys.washington.edu
}

\begin{abstract}
The proton radius puzzle is one of the most perplexing physics issues of recent times. The extremely precise extraction of the proton radius by Pohl et al. from the measured energy difference between the $\mathrm{P}$ and $\mathrm{S}$ states of muonic hydrogen disagrees with that extracted from electronic hydrogen. The newly extracted value of the proton radius is smaller than the previous one of CODATA (based mainly on electronic $\mathrm{H}$ ) by about $4 \%$ or 5.0 standard deviations. The three most likely possibilities for resolving the puzzle are (1) the electronic results are not quite as accurate as stated, (2) the muon-proton interaction is not the same as the electron-proton interaction, and (3) hadronic effects in the two-photon exchange term that are unimportant in electronic hydrogen are important in muonic hydrogen. This paper is concerned with the third possibility.
\end{abstract}

Xth Quark Confinement and the Hadron Spectrum

$8 Ð 12$ October 2012

TUM Campus Garching, Munich, Germany

\footnotetext{
*Speaker.

${ }^{\dagger}$ This work is supported in part by the USDOE .
} 


\section{The proton radius puzzle}

Pohl et al. [1] made a new measurement of the Lamb shift in muonic hydrogen. The article was a cover story in Nature under the popularized title of "Shrinking the Proton". Of course the proton did not shrink. It is simply that the extracted value of the radius is $0.84184(67) \mathrm{fm}$ which is smaller than the value obtained from electronic hydrogen $0.8768(69) \mathrm{fm}$ [2]. This larger value is (with increased error bars), also obtained from electron-proton scattering. The four percent reduction in the radius might seem inconsequential, but we shall see that this is not the case. Before proceeding, it is necessary to note the definition of the term proton radius that is used in the atomic physics community: $r_{p}^{2}=-6 \frac{d G_{E}}{d Q^{2}}\left(Q^{2}=0\right)$. This is the quantity that enters in the atomic physics experiments. However, this radius is not related to an integral over a rigorously-defined charge density of the proton $[3,4]$.

Before proceeding we should ask: how is it that atomic physics can be used to measure the proton radius and why should muonic hydrogen be more sensitive to this quantity than electronic hydrogen? Any sensitivity to proton structure depends on the probability that the lepton exists inside the volume of the proton. This probability depends on the ratio of the proton and atomic volumes: $\left(r_{p} / a_{B}\right)^{3} \sim\left(\alpha^{3} r_{p}\right)^{3} m^{3}$, which is proportional to the lepton mass to the third power. This factor is about 8 million times larger for the muon than for the electron. The factor of $\alpha^{3}$ causes the effects of proton structure to be small.

The Schroedinger and Dirac equations predict that the $2 S_{1 / 2}$ and $2 P_{1 / 2}$ states in hydrogen atoms are degenerate, so the splitting, the Lamb shift is important. Effects of the self-energy of the electron dominate the Lamb shift in electronic hydrogen and the vacuum polarization term dominates in muonic hydrogen, accounting for about $205 \mathrm{meV}$ of the total $\sim 206 \mathrm{meV}$ splitting. The effect of the finite size of the proton is mainly given by the term $\Delta E=\frac{2}{3} \pi \alpha|\phi(0)|^{2} r_{p}^{2}$, where $\phi(00$ is the $2 S$ wave function evaluated at the origin.

\subsection{Experimental Summary}

Pohl et al. use very precise pulsed laser spectroscopy "to measure a muonic Lamb shift of $49,881.88(76) \mathrm{GHz}$ ". The idea is that $1 \%$ of stopped muons from the PSI accelerator populate the $2 S$ state. The $2 S \rightarrow 2 P$ transitions are induced by a laser. The $2 P$ state decays via emission of an $E 1, \gamma$ ray which is detected in coincidence with the laser. The measured transition goes from the $2 S_{1 / 2}, F=1$ state to the $2 P_{3 / 2}, F=2$ state. Then various calculations are used to deduce the energy for the needed $2 S_{1 / 2} \rightarrow 2 P_{1 / 2}$ transition. These calculations were done long ago by many authors; see the discussion in [1], and parts have been repeated recently [5, 6] by several authors including us $[7,8,9]$. No significant changes in previously reported effects have been found. Something new is needed.

Pohl et al. used the various existing calculations to find $r_{p}=0.84184(67) \mathrm{fm}$, which "differs by 5.0 standard deviations from the CODATA value [2] of 0.8768(69) fm." This result implies that either the Rydberg constant has to be shifted by (4.9 standard deviations), or the calculations of the QED effects in atomic hydrogen or muonic hydrogen atoms are insufficient". The extracted proton radius is $4 \%$ smaller than previously measured. The Rydberg constant $R_{\infty} \equiv m_{e} e^{4} /\left(8 \varepsilon_{0}^{2} h^{3} c\right)$ ( $E_{\mathrm{Bohr}} \equiv R_{\infty} / n^{3}$ ) is known to 12 significant figures, so revising it by 4.9 standard deviations would be a spectacular step. 
The failure to understand how the proton radius can be different in muonic and electronic hydrogen is indeed a puzzle.

\subsection{Possible Resolutions}

There are various possibilities to resolve the proton radius puzzle:

- Either the muonic or electronic experiments are wrong, by a small amount larger than stated error bars

- QED bound states calculations are slightly wrong

- The muon interacts differently than electron

- Strong interaction effects appearing the two photon exchange term $[10,11]$ related to proton polarizability.

It is difficult to understand how the muonic experiment can be wrong. The authors state "The 1S-2S transition in $\mathrm{H}$ has been measured to $34 \mathrm{~Hz}$, that is, $1.4 \times 10^{-14}$ relative accuracy. Only an error of about 1,700 times the quoted experimental uncertainty could account for our observed discrepancy." Furthermore, the huge number of times the electronic experiments have been done make it seem unlikely there is an error there. However Pohl has suggested that several of the most accurate experiments are not independent, so the error bar may be artificially small. Moreover, several new experiments are underway. The possibility of an underestimate of the error is real. However, a 5 standard deviation shift in the Rydberg constant would cause trouble for QED because the existing calculations work so well. So far no one has found a significant error in the QED bound state calculations. Therefore we discuss the latter two effects.

\subsubsection{The muon interacts differently than the electron}

This is the most exciting possibility because electron-muon universality is a basic part of the Standard Model. Marciano, gave a talk at the INT during the summer of 2010 in which he explained that using a massive photon which only occurs in muonic interactions, thereby violating $\mu-e$ universality, would lead to huge matter effects in neutrino oscillations. These are too big by a factor of 10000. Barger et al. [12] "consider exotic particles that among leptons, couple preferentially to muons, and mediate an attractive nucleon-muon interaction. We find that the many constraints from low energy data disfavor new spin- -0 , spin- 1 and spin- 2 particles as an explanation." Brax and Burrage [13] obtain the same result for the scalar mesons that they study.

Batell, McKeen, and Pospelov [14] say that the recent discrepancy between proton charge radius measurements extracted from electron-proton versus muon-proton systems suggests the existence of a new force differentiating between lepton species. They identify a class of models with gauged right-handed muon number, which contains new vector and scalar force carriers at the $100 \mathrm{MeV}$ scale or lighter, that is consistent with observations. Such forces would lead to an enhancement by several orders-of-magnitude of the parity-violating asymmetries in the scattering of low-energy muons on nuclei. This is an exciting possibility. Note also that the assumed Lagrangian supplies a contribution that could resolve the current lack of understanding of the muon magnetic moment. 
Tucker-Smith \& Yavin [15] are optimistic about new force carriers solving the proton radius puzzle, stating the assumptions of Barger et al. are model-dependent. Calrson \& Rislow [16] also find that new physics with fine-tuned couplings may be entertained as a possible explanation of the proton radius puzzle.

\section{Experimental analysis}

The proton radius is extracted from one measured transition energy, $\xi=206.2949(32)$, equated to a sum of contributions:

$$
\xi=209.9779(45)-5.2262 r_{p}^{2}+0,0347 r_{p}^{3} \mathrm{meV} .
$$

Solving the cubic equation for $r_{p}$ leads to the stated result. To explain the puzzle, one might start by analyzing the largest number on the right hand side of the equation. One needs to increase the number $209.9779 \mathrm{meV}$ by $0.31 \mathrm{meV}=3.1 \times 10^{-10} \mathrm{MeV}$. This tiny number causes big trouble.

\subsection{Why proton polarizability?}

Proton structure effects are proportional to the square of the wave function at the origin, proportional to the lepton mass to the third power. Despite this strong dependence, it is easy to see that any effect large enough to yield $0.31 \mathrm{meV}$ for muonic hydrogen will destroy the spectacular agreement between theory and experiment for electronic hydrogen. On the other hand, the effects of the proton polarizability, shown schematically in Fig. 1, introduce, via the lepton propagator an additional factor of the lepton mass. The polarizability graph is proportional to $\alpha^{5} \mathrm{~m}^{4}$ and is capable of accounting for the proton radius puzzle in muonic hydrogen without influencing the electronic hydrogen results.

Our first idea [10] was based on the fact that the intermediate proton is off its mass shell. Therefore its form factor is not the same as in free space and we postulated a model form factor that could account for the needed $0.31 \mathrm{meV}$. This model, although satisfying current conservation, was criticized for not satisfying constraints arising from low energy theorems [17]. We later found that It is possible to construct an off-shell proton form factor that is consistent with constraints from current conservation and low-energy theorems, while also providing a contribution to the Lamb shift that might potentially resolve the proton radius puzzle in muonic hydrogen. However, observations of quasi-elastic scattering limit the overall strength of the off-shell form factors to values that correspond to small contributions to the Lamb shift [18]

But there is a way to proceed that satisfies all of the constraints, finds an energy shift in accord with the muonic data, and is testable in a separate experiment [11].

\subsection{Previous estimates and their uncertainty}

Because our result [11] seems to be at variance with previous estimates it is necessary to discuss the relationship with the conventional approach [19,20]. The energy shift due to polarization $\Delta E$ is given by:

$$
\Delta E \propto \alpha^{5} m^{3} \int \frac{d^{4} q}{q^{4}} T^{\mu v} L_{\mu v}
$$




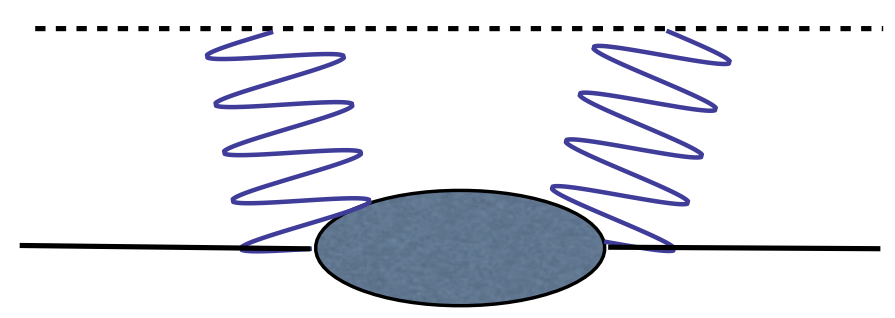

Figure 1: Direct two-photon exchange graph corresponding to polarizability effects. The dashed line denotes the lepton; the solid line, the nucleon; the wavy lines photons; and the ellipse the intermediate states.

where $T^{\mu v}$ is the forward virtual-photon proton scattering amplitude, and $L_{\mu v}$ is the lepton tensor. The quantity $T^{\mu v}$ has a field-theoretic definition:

$$
T^{\mu v}(q, P)=-i \int d^{4} x e^{i q \cdot x}\left\langle P\left|T\left(j^{\mu}(x) j^{\mu}(0)\right)\right| P\right\rangle,
$$

where averaging over the proton spin is understood, $P$ is the proton four-momentum, $j^{\mu}$ is the electromagnetic current operator and gauge invariance tells us that there are two independent dynamical quantities:

$$
T^{\mu v}(q, P)=-\left(g^{\mu v}-\cdots\right) T_{1}\left(q \cdot P, q^{2}\right)+\left(P^{\mu}-\cdots\right)\left(P^{v}-\cdots\right) T_{2}\left(q \cdot P, q^{2}\right) .
$$

One would like to evaluate Eq. (2.3) by using completeness and known matrix elements; however this can not be done. Instead one uses the fact that $\operatorname{Im}\left[T_{1,2}\right] \propto W_{1,2}$ the measured proton structure functions. The real part, which is relevant here, can be obtained from the imaginary part using dispersion relations. Thus one is using Cauchy's theorem plus data, a procedure that should be rock solid. What could go wrong?

The main problem is that $\operatorname{Im}\left[T_{1}\right]$ falls too slowly with $v \equiv q \cdot P$ for large values of $v$, so that using a subtracted dispersion relation is necessary to determine $T_{1}$,. Thus a subtraction function, defined as $\bar{T}_{1}\left(0, Q^{2}\right)$ is needed at $q \cdot P=0$ for all values of $q^{2}=-Q^{2}$. Indeed Hill \& Paz [21] argued that the uncertainty is an order of magnitude larger than stated. I argue that the uncertainty is two orders of magnitude larger than stated.

The contribution to the Lamb shift that is caused by $\bar{T}_{1}\left(0, Q^{2}\right)$ is denoted as $\Delta E^{\text {subt }}$ and is given by $[19,22,20,17]$

$$
\Delta E^{\text {subt }}=\frac{\alpha^{2}}{m} \phi^{2}(0) \int_{0}^{\infty} \frac{d Q^{2}}{Q^{2}} h\left(Q^{2}\right) \bar{T}_{1}\left(0, Q^{2}\right)
$$

where $\phi^{2}(0)=\frac{\alpha^{3} m_{r}^{3}}{8 \pi}$ for the $2 \mathrm{~S}$ state with $m_{r}$ as the lepton reduced mass, and

$$
h(t)=\left(1-\frac{t}{2 m^{2}}\right)\left(\left(1+\frac{4 m^{2}}{t}\right)^{1 / 2}-1\right)+1 .
$$

The function $h(t)$ is monotonically falling, approaching $2 \mathrm{~m} / \sqrt{t}$ for small values of $t$, and falling as $2 m^{2} / t$ large values of $t$. Low energy theorems tell us that $\bar{T}_{1}\left(0, Q^{2}\right) \sim Q^{2}$ for small values of $Q^{2}$. If such behavior is used in Eq. (2.6), the integral would diverge logarithmically. 
Various authors $[19,20,22]$ have used the relation

$$
\bar{T}_{1}\left(0, Q^{2}\right)=\frac{\beta_{M}}{\alpha} Q^{2} F_{\text {loop }}\left(Q^{2}\right),
$$

where $\beta_{M}$ is the known magnetic polarizability of the proton, and $F_{\text {loop }}\left(Q^{2}\right)$ is a form factor that is inserted to make the integral expression Eq. (2.6) converge.

Birse \& McGovern [23] evaluate terms up to fourth-order in chiral perturbation theory to find

$$
\bar{T}_{1}^{B M}\left(0, Q^{2}\right) \simeq \frac{\beta_{M}}{\alpha} Q^{2}\left(1-\frac{Q^{2}}{M_{\beta}^{2}}+\mathscr{O}\left(Q^{4}\right)\right) \rightarrow \frac{\beta_{M}}{\alpha} Q^{2} \frac{1}{\left(1+\frac{Q^{2}}{2 M_{\beta}^{2}}\right)^{2}},
$$

with $M_{\beta}=460 \pm 50 \mathrm{MeV}$.

I proceeded to exploit the uncertainty arising from the unknown high $Q^{2}$ behavior, by postulating forms with stronger contributions at very large values of $Q^{2}$ that fall more slowly using two different methods.

First, I chose [11] a form of $F_{\text {loop }}\left(Q^{2}\right)$ that is consistent with the constraints introduced by Birse \& McGovern [23]. This is done by postulating a term that begins at order $Q^{6}$ in Eq. (2.7), such as

$$
F_{\text {loop }}\left(Q^{2}\right)=\frac{Q^{4}}{M_{\gamma}^{4}} \frac{1}{\left(1+a Q^{2}\right)^{3}} .
$$

This ignores their $Q^{2}$ and $Q^{4}$ terms (which produce negligible results), but supplies a new contribution arising from the large $Q^{2}$ regime of the integrand of Eq. (2.5). Then we find

$$
\Delta E^{\text {subt }} \approx \frac{3}{2} m \alpha \phi^{2}(0) \frac{\beta_{M}}{M_{\gamma}^{4} a^{2}} .
$$

Using $a=0.0078 /\left(4 m_{\mu}^{2}\right)=0.177 \mathrm{GeV}^{-2}, 1 / a=5.65 \mathrm{GeV}^{2}$ leads to the necessary Lamb shift of $0.31 \mathrm{meV}$. Indeed, a relatively large number must appear to obtain the necessary contribution, but no fundamental principles have been violated.

Another approach is to use effective field theory, EFT. In this case, one handles the would be logarithmic divergence by using dimensional regularization and fixing an unknown finite piece to the only piece of data-the $0.31 \mathrm{meV}$ needed to account for the proton radius puzzle. The corresponding contribution to the Lamb shift is given by

$$
\Delta E^{D R}=\alpha^{2} m \frac{\beta_{M}}{\alpha} \phi^{2}(0)(\lambda+5 / 4) .
$$

Setting $\Delta E^{D R}$ to $0.31 \mathrm{meV}$ in the above equation requires that $\lambda=769$ which seems like a large number. However, $\beta_{M}$ is extraordinarily small due to a cancellation between paramagnetic effects of an intermediate $\Delta$ and diamagnetic effects of the pion cloud [24]. The natural units of polarizability are $\frac{\beta_{M}}{\alpha} \sim 4 \pi / \Lambda_{\chi}^{3}$, [25] where $\Lambda_{\chi} \equiv 4 \pi f_{\pi},\left(f_{\pi}\right.$ is the pion decay constant $)$. Then the scattering amplitude corresponding to Fig. 1 becomes

$$
\mathscr{M}_{2}^{D R}=i 3.95 \alpha^{2} m \frac{4 \pi}{\Lambda_{\chi}^{3}} \bar{u}_{f} u_{i} \bar{U}_{f} U_{i}
$$


The coefficient 3.95 is of natural size. Thus standard EFT techniques result in an effective leptonproton interaction of natural size that is proportional to the lepton mass.

The present results, Eq. (2.10) and Eq. (2.11) represent an assumption that there is a leptonproton interaction of standard-model origin, caused by the high-momentum behavior of the virtual scattering amplitude, that is sufficiently large to account for the proton radius puzzle. Fortunately, our hypothesis can be tested in an upcoming low-energy $\mu^{ \pm} p, e^{ \pm} p$ scattering experiment [26] planned to occur at PSI.

\section{Lepton Proton Scattering at Low Energies}

Our aim is to provide a prediction for the PSI experiment. It is well-known that two-photon exchange effects in electron-proton scattering are small at low energies. Our effect $\mathscr{M}_{2}$ provides a two-photon exchange contribution to the lepton-proton scattering amplitude that interferes with the one-photon exchange term. Our contact interaction is proportional to the lepton mass, so it could provide a measurable effect for muon-proton scattering but be ignorable for electron-proton scattering. We investigated the two consequences of using form factors (FF) and EFT. The results are in Fig. 2 of Ref. [11]. Effects of between 2 and 4\% (depending on scattering angle) are found. The size of the effect should be detectable within the expected sub-1\% accuracy of the PSI experiment.

\section{Summary and Discussion}

The findings of this paper can be summarized with a few statements:

- A logarithmic divergence in the integrand that determines the value of $\Delta E^{\text {subt }}$ causes the uncertainty in the evaluation to be large enough to account for the proton radius puzzle.

- The logarithmic divergence can be controlled using a form factor or the dimensional regularization procedure.

- Either method can be used to account for the proton radius puzzle and predict an observable effect of a few percent for low energy $\mu-p$ scattering.

The literature poses several explanations for the proton radius puzzle: The electronic-hydrogen experiments might not be as accurate as previously reported, $\mu-e$ universality might be violated, and that a strong interaction effect entering in the two-photon exchange diagram is important for muonic hydrogen, but not for electronic hydrogen. It is beyond the scope of the present paper to argue for the unique correctness of any one of these ideas. The effect discussed here is large enough to be testable in an independent experiment.

\section{References}

[1] R. Pohl, A. Antognini, F. Nez, F. D. Amaro, F. Biraben, J. M. R. Cardoso, D. S. Covita and A. Dax et al., Nature 466, 213 (2010).

[2] P. J. Mohr, B. N. Taylor and D. B. Newell, Rev. Mod. Phys. 80, 633 (2008) [arXiv:0801.0028 [physics.atom-ph]]. 
[3] G. A. Miller, Phys. Rev. Lett. 99 (2007) 112001 [arXiv:0705.2409 [nucl-th]].

[4] G. A. Miller, Ann. Rev. Nucl. Part. Sci. 60 (2010) 1 [arXiv:1002.0355 [nucl-th]].

[5] U. D. Jentschura, Phys. Rev. A 84 (2011) 012505 [arXiv:1107.1737 [physics.atom-ph]].

[6] U. D. Jentschura, Annals Phys. 326 (2011) 500 [arXiv:1011.5275 [hep-ph]]. U. D. Jentschura, Annals Phys. 326 (2011) 516 [arXiv:1011.5453 [hep-ph]].

[7] I. C. Cloet and G. A. Miller, Phys. Rev. C 83, 012201 (2011)

[8] J. D. Carroll, A. W. Thomas, J. Rafelski and G. A. Miller, Phys. Rev. A 84 (2011) 012506 [arXiv:1104.2971 [physics.atom-ph]].

[9] J. D. Carroll, A. W. Thomas, J. Rafelski and G. A. Miller, arXiv:1108.2541 [physics.atom-ph].

[10] G. A. Miller, A. W. Thomas, J. D. Carroll, J. Rafelski, Phys. Rev. A 84 (2011) 020101

[11] G. A. Miller, Phys. Lett. B. 718 (2013) 1078 arXiv:1209.4667 [nucl-th].

[12] V. Barger, C. -W. Chiang, W. -Y. Keung and D. Marfatia, Phys. Rev. Lett. 106, 153001 (2011) [arXiv:1011.3519 [hep-ph]].

[13] P. Brax and C. Burrage, Phys. Rev. D 83, 035020 (2011) [arXiv:1010.5108 [hep-ph]].

[14] B. Batell, D. McKeen and M. Pospelov, Phys. Rev. Lett. 107 (2011) 011803 [arXiv:1103.0721 [hep-ph]].

[15] D. Tucker-Smith and I. Yavin, Phys. Rev. D 83, 101702 (2011) [arXiv:1011.4922 [hep-ph]].

[16] C. E. Carlson and B. C. Rislow, Phys. Rev. D 86 (2012) 035013 arXiv:1206.3587 [hep-ph].

[17] C. E. Carlson and M. Vanderhaeghen, arXiv:1109.3779 [physics.atom-ph].

[18] G. A. Miller, A. W. Thomas and J. D. Carroll, Phys. Rev. C 86 (2012) 065201 arXiv:1207.0549 [nucl-th].

[19] K. Pachucki, Phys. Rev. A 60, 3593 (1999).

[20] C. E. Carlson and M. Vanderhaeghen, Phys. Rev. A 84, 020102 (2011) [arXiv:1101.5965 [hep-ph]].

[21] R. J. Hill and G. Paz, Phys. Rev. Lett. 107, 160402 (2011)

[22] A. P. Martynenko, Phys. Atom. Nucl. 691309 (2006) 1309 [hep-ph/0509236].

[23] M. C. Birse and J. A. McGovern, Eur. Phys. J. A 48 (2012) 120 arXiv:1206.3030 [hep-ph].

[24] A. W. Thomas and W. Weise, "The Structure of the Nucleon," Berlin, Germany: Wiley-VCH (2001) $389 \mathrm{p}$

[25] M. N. Butler and M. J. Savage, Phys. Lett. B 294 (1992), 369

[26] PSI proposal R-12-01.1, "Studying the Proton "Radius" Puzzle with mu-p Elastic Scattering", J. Arrington et al. 